\title{
ALFABETISMO CIENTÍFICO, MISIÓN DE LA UNIVERSIDAD Y CIUDADANÍA: IDEAS PARA SU CONSTRUCCIÓN EN LOS PAÍSES EN VÍAS DE DESARROLLO
}

\author{
Francisco López Segrera* \\ Cristian Parker Gumucio**
}

Recebido em: 10 de novembro de 2008

Aprovado em: 15 de março de 2009

\begin{abstract}
*Dr. en Estudios latinoamericanos (París VIII, Sorbonne). Vicerrector del Instituto de Relaciones Internacionales de Cuba (ISRI) 1974-1988. Trabajó en UNESCO entre 1994 y 2002, donde se desempeñó como Consejero Regional de Ciencias Sociales y Director del Instituto Internacional para la Educación Superior en América Latina y el Caribe (IESALC). Sus últimos libros publicados sobre educación superior son Escenarios Mundiales de la Educación Superior. CLACSO, 2006; y La educación superior en América Latina y el Caribe 2008 (Editor junto a Colin Brock y José Dias Sobrinho), Avaliacao, julio de 2008. Es miembro del Foro de UNESCO sobre Educación Superior, investigación y conocimiento. Miembro del Grupo de Trabajo de CLACSO sobre Universidad y Sociedad. Actualmente trabaja como Asesor Académico de la Global University Network for Innovation y Editor de La Educación Superior en el Mundo. Email: francisco. lopez-segrera@upc.edu.

** Chileno, sociólogo, Dr. en Sociología (UC de Lovaina); Profesor Titular de la Universidad de Santiago de Chile; Director del Instituto de Estudios Avanzados de la misma Universidad; Director del Magíster en Política Exterior, (Convenio USACH - Academia Diplomática de Chile). Profesor del Doctorado en Estudios Americanos (USACH). Profesor de la Academia Diplomática de Chile en la cátedra de metodología de Investigación . Profesor del Magíster en Historia Militar y Pensamiento Estratégico de la Academia de Guerra del Ejército de Chile en la cátedra de Metodología de la Investigación Científica. E-mail: cparker@ lauca.usach.cl
\end{abstract}

Resumen: El presente artículo presenta un análisis del nuevo concepto de alfabetismo científico como competencia requerida para el ejercicio de la ciudadanía en el siglo XXI y como complemento y ampliación del alfabetismo tradicional. Ya no basta con enseñar a leer y escribir, es necesario también hacer realidad la educación permanente para todos y el acceso universal a las Tics en un marco de pertinencia e inclusión social.

Palabras-clave: Educación superior. Alfabetismo centífico. Misión de la Universidad. Ciudadanía.

\section{SCIENTIFIC LITERACY, MISSION OF THE UNIVERSITY AND CITIZENSHIP: IDEAS FOR THEIR CONSTRUCTION IN DEVELOPING COUNTRIES}

Abstract: The present article is an analysis of the new concept of scientific literacy as a requirement for the
exercise of citizenship in the 21st century and as complement and extension of traditional literacy.
It is no longer enough to teach how to read and write, it is necessary also to make lifelong education
for all and the universal access to the ICTs a reality in a frame of relevancy and social inclusion.

Keywords: Higher education. Scientific literacy. Mission of the University. Citizenship. 


\section{Introducción}

El "alfabetismo científico" - que implica el alfabetismo digital, visual, tecnológico, electrónico, informaciónal, científico, tecnológico, etc., pero que no se agota en ellos -, es una competencia global requerida para el ejercicio de la ciudadanía en el siglo XXI, la cuál debe entenderse como una "ciudadanía integral" y no sólo en términos relativos al ejercicio de derechos cívicos, económicos y sociales.

Fortalecer el alfabetismo científico en los países en vías de desarrollo frente al riesgo de dominio tecnocrático, implica construir la equidad y la ciudadanía en forma paralela. Solo así podremos hablar de un alfabetismo científico pertinente, mensurable mediante indicadores ad hoc. La investigación científica orientada a los problemas de los países en desarrollo y las universidades nacionales de estos países, puede desempeñar un papel clave en la construcción de este nuevo paradigma - que integre saber ancestral y ciencia, conocimiento global y local- para lo cuál ofreceremos algunas recomendaciones.

\section{La nueva dimensión del alfabetismo y del analfabetismo}

La tercera revolución industrial, la de las TIC, en un entorno globalizado de acelerado progreso científico-tecnológico, a dado lugar a un nuevo concepto de analfabetismo que implica en esencia el desconocimiento, por un lado, de los principios básicos de la ciencia actual y ,por otro, la incapacidad de acceso, comprensión y uso de la cultura digital. Afecta en especial a personas adultas y a colectivos sociales desfavorecidos tanto en países desarrollados como en desarrollo, aunque son estos últimos los que tienen una mayor cantidad de analfabetos en el sentido tradicional y en el moderno. Es un factor de desigualdad ciudadana en tanto que desigualdad económica, política y social. Hasta ahora ser alfabeto era tener la destreza necesaria para saber leer y escribir textos. Hoy ser alfabeto implica saber manejar adecuadamente las TIC y conocer además el latín del mundo moderno, esto es, el idioma inglés. Sin saber buscar y seleccionar la información, interpretarla críticamente, trabajar en red y comunicarse y expresarse a través de las TIC con imagen, sonido, e hipertextos un ciudadano del siglo XXI no puede considerarse alfabetizado.

La alfabetización tradicional tenía como objetivos "formar ciudadanos cultos, con capacidad crítica y poder para transformar la realidad" (FREIRE, 1999, p. 50). La nueva alfabetización (conocimiento de los principios básicos 
de la ciencia, de las TIC + dominio del idioma inglés) tiene los mismos objetivos, pero requiere nuevos conocimientos. Un concepto a medio camino entre el analfabetismo tradicional, esto es, incapacidad para leer y escribir textos, y el nuevo alfabetismo, es el analfabetismo funcional propio de las personas iletradas que, aunque formalmente han sido alfabetizadas, no pueden entender un mensaje sencillo, debido a que su mecanismo lector no es capaz de discriminar las ideas principales de las ideas secundarias ni comprender a plenitud los conceptos que lee debido a su carencia de vocabulario.

La nueva alfabetización no implica solamente lo señalado más arriba. ¿Puede considerarse alfabetizado una persona que tenga un conocimiento de las TIC que no rebase la Web 1.0? ¿ No es acaso necesario dominar la Web 2.0 para considerarse alfabeto en el nuevo sentido moderno? Solo queremos enfatizar con estas preguntas el carácter dinámico del concepto de alfabetismo científico, que se suele aplicar a la nueva alfabetización para distinguirla de la tradicional.

Podemos ir ahora a ver con mayor profundidad ciertos aspectos del alfabetismo científico que suele contraponerse al alfabetismo tradicional en tanto que nuevo alfabetismo.

Nadie duda de que la divulgación de los últimos resultados de la investigación, a menudo sobre experimentos y teorías complejas, sea poco practicable en un contexto de analfabetismo científico de base. Las carencias de cultura científica de la ciudadanía esta referida a aspectos muy básicos, sin los cuales no es posible entender el trabajo científico, por lo que la ciencia y sus aplicaciones se convierten en algo incomprensible e irracional, que colinda con la magia. La ciencia continúa considerándose como práctica exclusiva de los especialistas, "como algo no constitutivo de la cultura y a cuya comprensión elemental se renuncia a priori".

La National Science Education Standards (NRC, 1996) define el concepto de analfabetismo científico del siguiente modo: "Alfabetismo científico significa que una persona puede preguntar, encontrar o determinar respuestas a preguntas derivadas de la curiosidad acerca de las experiencias diarias. Significa que una persona tiene la habilidad para describir, explicar, y predecir fenómenos naturales. Implica que una persona pueda identificar aspectos científicos que soportan las decisiones de tipo local o nacional y exprese opiniones al respecto sustentándose tanto científica como tecnológicamente".

Ser un alfabeto científico implica estar familiarizado con los conocimientos básicos y fundamentales de la ciencia actual, que en orden de importancia son los siguientes (TREFIL, 1996 apud CABRAL, 2001): 


\author{
Causalidad/orden \\ Energía/entropía \\ Electricidad/magnetismo \\ El átomo \\ Enlace químico/reacciones químicas \\ Selección natural/evolución \\ Biología celular/bioquímica \\ Código genético/medicina molecular \\ Tectónica de placas \\ El Big Bang/origen del universo \\ Estrellas y galaxias \\ Genética mendeliana \\ Propiedades de los materiales \\ Ciclos terrestres \\ Ecosistemas \\ Relatividad \\ Mecánica cuántica
}

Bajo esta misma tónica, un documento de Science for All Americans (AAAS, 1989) indica que los conocimientos básicos que debe poseer un alfabeto científico son:

La estructura y evolución del universo, con un énfasis en la similitud de materiales y fuerzas encontrado en todos lados, el poder de unos principios generales (tales como la gravitación universal y la conservación de la energía).

Las características generales del planeta Tierra, incluyendo su localización, movimientos, origen y recursos; la dinámica por la cual su superficie es formada y transformada; el efecto de los organismos vivientes sobre su superficie y atmósfera; y cómo sus tierras, ríos y océanos, clima y recursos tienen una influencia sobre y cómo viven las personas, y cómo se ha desarrollado la historia humana.

Los conceptos básicos relacionados con la materia, energía, fuerza y movimiento, con un énfasis sobre su uso en modelos para explicar un vasto y diverso arreglo de fenómenos naturales desde el nacimiento de las estrellas hasta el comportamiento de las células.

La rica diversidad de los organismos de la Tierra y la sorprendente similitud en la estructura y funciones de sus células, la dependencia de las especies sobre otras y sobre el ambiente físico, y el flujo de materia y energía a través de los ciclos de la vida. 
La evolución biológica, concepto basado en la extensa evidencia geológica y molecular, como una explicación de la diversidad y similitud de las formas de vida y como un principio organizador central para toda la biología.

La estructura básica y funcionamiento del cuerpo humano, visto como un sistema de células y órganos que sirven a las funciones de obtención de energía a partir de los alimentos, protección contra daños, coordinación interna y la reproducción.

La naturaleza de las tecnologías, incluyendo la agricultura, con un énfasis tanto en la revolución agrícola de tiempos antiguos como en los efectos de la productividad agrícola del siglo XX con el uso de tecnologías biológicas y químicas; la adquisición, procesamiento, y uso de materiales y energía, con particular atención a la Revolución Industrial y a la actual revolución en la manufactura basada en el uso de computadoras; y el procesamiento de la información y las comunicaciones, con énfasis en el impacto de computadoras y comunicaciones electrónicas en la sociedad contemporánea. ${ }^{1}$

Para tener una dimensión de la necesidad de programas para lograr el alfabetismo científico tanto en países desarrollados como en desarrollo basta consultar en la Web las encuestas en EE.UU. de la National Science Foundation o bien las llevadas a cabo en México u otros países. En una encuesta realizada en los Estados Unidos por la National Science Foundation, el 50\% de los adultos desconocía que la Tierra girase alrededor del sol y que tardara un año en hacerlo; sólo el 21\% puede definir al ADN; nada más el $9 \%$ sabe lo que es una molécula; el 63\% ignoraba que los dinosaurios se extinguieron antes de la desaparición del hombre; el 75\% no sabía que los antibióticos sólo matan a las

1 Se suelen incluso elaborar diversos Test para medir el grado de alfabetismo científico.

\section{TEST 1:}

¿Qué es la entropía según la física moderna?

¿Cuáles son los mecanismos de la evolución?

¿Qué sabes del código genético?

¿Puedes describir el Big Bang o comienzo del universo?

¿Qué es la mecánica cuántica?

¿Por qué son importantes los experimentos de Mendel con sus guisantes o chicharitos?

TEST 2:

"Tomates modificados por ingeniería genética rompen récord de producción"

"Japón toma la delantera en la carrera de superconductores"

"Se envía sonda a Marte equipada con tres espectrofotómetros para analizar su superficie"

"Científicos descifran el 99\% del código genético humano"

"Es descubierto el quasar más distante de la tierra"

\section{TEST 3:}

¿Cuánto tiempo tarda la Tierra en girar en torno al sol?

¿Qué es el ADN?

¿Qué es una molécula?

¿Los dinosaurios se extinguieron antes o después de que apareciera el hombre?

¿Son efectivos los antibióticos para aniquilar a los virus? 
bacterias y no a los virus; 25 millones de estadounidenses no pudieron localizar a los EE.UU. en un mapa del mundo. Por otra parte, en otra encuesta aplicada a principios de 1999 en el Distrito Federal, en México, los resultados también fueron similares: el 57.2\% supo que las plantas de la Tierra evolucionaron; sólo el $48 \%$ dijo que la temperatura del cuerpo varía entre 36 y 37 grados Celsius; $77 \%$ cree que la astrología modifica sus vidas; $38 \%$ cree en la existencia de brujas; y $24.5 \%$ cree que existen los vampiros.

\section{Alfabetismo científico y ciudadanía}

En la sociedad de la información, a los ciudadanos y ciudadanas ya no les bastará con estar alfabetizados en un sentido tradicional, en la cultura letrada; deben también estar alfabetizados en un tipo especial de cultura letrada, la de los textos electrónicos, y han de estar alfabetizados, esto es, ser competentes, en las tecnologías digitales, en los lenguajes audiovisuales, en el manejo de la información, y además dominar el idioma inglés y conceptos esenciales de ciencia y tecnología.

Ser ciudadano implica ser miembro de una comunidad política. La condición de miembro de dicha comunidad se considera ciudadanía, e implica una serie de deberes y derechos. La ciudadanía ha sido definida como "el derecho y la disposición de participar en una comunidad, a través de la acción autorregulada, inclusiva, pacífica y responsable, con el objetivo de optimizar el bienestar público."

El concepto de ciudadanía ha cambiado a lo largo de la historia haciéndose cada vez menos excluyente, al menos en lo formal. En la democracia ateniense, sólo eran considerados ciudadanos los hombres libres varones, y por ello las mujeres y los esclavos tenían privado todo tipo de participación en la vida política.

En las democracias actuales, tienen la condición de ciudadanos todos los hombres y mujeres mayores de edad, aunque cada vez se maneja más el concepto de ciudadanía formal, en el sentido de que no todos los ciudadanos gozan por igual de los derechos políticos, económicos y sociales, de ahí un creciente fenómeno de abstencionismo político y electoral en los países tanto desarrollados como en desarrollo. En los países en desarrollo y en América Latina y el Caribe en especial se ha desarrollado el concepto de "exclusión social", "apartheid social" y "ciudadanía negada" para destacar el hecho de que los pobres son solo ciudadanos formales, en tanto no tienen acceso a los beneficios de la democracia. Se ven privados de derechos sociales ciudadanos básicos como la 
salud y la educación, de derechos económicos como el de acceder a un empleo e incluso de derechos políticos y jurídicos solo accesibles en términos reales a las capas de la población que no viven en la pobreza.

Si bien algunos datos apuntan a una cierta disminución de la pobreza en la región desde 2003, en estrecha relación con el crecimiento económico que se ha producido en ella debida al alza de los precios de ciertas materias primas y a la emergencia de gobiernos con políticas sociales al respecto, la desigualdad y la pobreza siguen siendo importantes factores que limitan el crecimiento Según CEPAL, Latinoamérica es la región más desigual del mundo y la más rezagada en términos de equidad. Esto se hace evidente en las grandes diferencias entre los sectores de más altos y de menores ingresos. Los pobres en América Latina y el Caribe son el 40,6\% de la población, algo más de 213 millones de personas. El porcentaje de personas que se estima viven en la pobreza extrema es de 100 millones, lo que implica que el 18\% de los "ciudadanos" viven en la pobreza extrema.

Esta profunda desigualdad económica y social se expresa en desigualdad educativa y es un factor que tiende a reforzar el círculo de la pobreza. El porcentaje de personas analfabetas se sitúa en torno a las 34 millones de personas, casi el 10\% de la población. Además, el 40\% de personas jóvenes y adultas, lo que supone cerca de 110 millones de personas, no han terminado sus estudios de educación primaria. Estudios recientes señalan que el porcentaje de alumnos que completan la educación secundaria es cinco veces superior entre aquellos que se encuentran en el quintil más rico de ingresos familiares que aquellos que se encuentran en el quintil más pobre, Mientras que el 23\% de los primeros terminan la educación superior, sólo el 1\% de los más pobres lo consiguen. El promedio de escolarización en el quintil superior es de 11,4 años mientras que en el quintil inferior de ingresos es de 3,1 años. Estas diferencias en los años de estudio tienen una clara repercusión en el acceso al mercado laboral y en las posibilidades de ingresos económicos posteriores. La relación entre pobreza, oportunidades educativas y mantenimiento en la situación de pobreza es estrecha, lo que pone de relieve las enormes dificultades para favorecer la movilidad social en Latinoamérica. Si bien la tasa de matrícula universitaria promedio en la región ha pasado de $17 \%$ a principios de los 90 s a cerca del $32 \%$ en la actualidad, la población de bajos recursos tiende a resultar excluida de la educación superior pública por haber asistido a escuelas de menor calidad, lo que les impide aprobar los exámenes de selección. Muchos van a ingresar en las universidades de menor calidad del sector privado. En Brasil el 74\% de los alumnos matriculados en universidades pertenecen al quintil 1 el más elevado 
y sólo un 4\% al menos elevado; en México la proporción es de 58 y 6; en Chile de 65 y 8; y en Ecuador de 42 y 6. (LOPEZ SEGRERA, 2008)

Para lograr una educación permanente para todos como postula UNESCO, para construir el alfabetismo científico como una dimensión clave de la ciudadanía, es necesario: el acceso de todos los alumnos a una educación de calidad, el logro de un modelo educativo orientado hacia el ejercicio de la ciudadanía, y fortalecer las instituciones de educación básica y superior a nivel mundial, y en especial en los países en desarrollo.

En un texto reciente se afirma lo siguiente:

El concepto de alfabetismo cientifico ha sido ampliamente debatido y si bien hay cierto consenso en lo que refiere a algunos de sus contenidos básicos, hay también diversas interpretaciones y controversia. Recientemente, más allá de una primera aproximación que ligaba este concepto a la enseñanza de las ciencias naturales, se está aceptando, cada vez más una concepción amplia. Esta concepción comprensiva deriva de la preocupación existente en medios académicos, internacionales y gubernamentales, por apuntar hacia una formación integral del futuro ciudadano en la cual el conocimiento y el espíritu crítico-científico no deben faltar. (PARKER, 2008, p. 5)

La desaparición del analfabetismo no supone únicamente que las personas adultas tengan la capacidad de comprender un texto y de escribirlo. Es también necesario superar el analfabetismo digital, el analfabetismo idiomático y el analfabetismo cívico y alcanzar las capacidades que constituyen los objetivos de la educación básica. La erradicación de analfabetismo exige una educación que garantice a todas las personas el ejercicio de la ciudadanía. Esto implica garantizar un porcentaje de equidad social sin el cuál no puede existir la educación permanente para todos, prerrequisito para construir el alfabetismo científico y ciudadano. En esta importante cuestión, en la garantía de una educación para la ciudadanía, se encuentra el segundo gran objetivo de los sistemas educativos, tras haber logrado el primero de ellos, esto es, el acceso universal. No se trata sólo de educar sobre la ciudadanía, es decir, sobre las normas y valores cívicos propios de las sociedades democráticas, ni tampoco de educar en y para la ciudadanía, a través del ejercicio en la escuela de valores democráticos, participativos, solidarios y de convivencia. Consiste, además, en preparar a todos los alumnos para que puedan integrarse de forma activa y adecuada en la sociedad, ampliar sus conocimientos, adaptarse a los cambios políticos, económicos, culturales, sociales y laborales y poder disponer de la palabra y de la acción con las que ejercer sus derechos y deberes como personas 
libres. Desde esta visión y perspectiva, toda la enseñanza ha de orientarse a la formación de ciudadanos competentes, libres, responsables y solidarios. En esta tarea, UNESCO, al igual que la OEI, ha tenido un papel activo para apoyar esfuerzos, relacionar experiencias y contribuir a que los países tengan presente que "la ciudadanía nacional se prolonga en la ciudadanía iberoamericana y en la ciudadanía mundial.” (MARCHESI, 2007, p. 14)

Esto implica "avanzar en formar una nueva cultura científica que incluya una necesaria formación ciudadana.” (PARKER, 2008, p. 9)

\begin{abstract}
Se trata de un alfabetismo de nuevo cuño que debe incorporar un renovado espíritu científico (no reductivista) con una visión más amplia de ciudadanía, que la defina más allá del ejercicio de los derechos clásicos, e incorpore también aspectos vinculados a los nuevos tipos de conocimientos (científico-técnicos) que afectaran las decisiones fundamentales y estratégicas de la evolución futura de las sociedades en procesos de globalización. Sólo de esta manera se puede garantizar en forma simultánea: incremento de capital humano especializado, desarrollo científico-tecnológico nacional, participación democrática, desarrollo sustentable y control frente al riesgo del dominio tecnocrático.
\end{abstract}

El riesgo de emergencia de nuevos fenómenos de analfabetismo e iletrismo es elevado, teniendo en cuenta el escenario en el que se formulan los objetivos del alfabetismo científico. Existe un "apartheid educacional" que afecta a la sexta parte de los 6,600 millones de habitantes del planeta, los excluidos de las TIC. Según UNESCO, a fines del 2005, cerca de 800 millones de personas aún no sabían leer ni escribir. La mayor parte de esta cifra son mujeres y viven en medios rurales, lo que compromete el futuro de millones de niños sin escolarizar. Las regiones con una situación más preocupante son los estados árabes, el África Subsahariana y Asia del Sur y del Oeste. Los datos de la UNESCO señalan que en estas zonas los índices de alfabetización son del 60\%, frente al 99\% aproximadamente en los países de la Organización para la Cooperación y el Desarrollo Económico (OCDE). De acuerdo con cifras de la UNESCO del 2008, en el mundo hay 774 millones de analfabetos, es decir, uno de cada cinco adultos en el planeta no sabe leer ni escribir. Además, 110 millones de jóvenes no han concluido su educación primaria. En América Latina se estima que 35 millones de personas son analfabetas. De ellas, 55 por ciento son mujeres. Existe, por otra parte, como muestra el Índice de Desarrollo Humano del PNUD (IDH) una estrecha correlación entre pobreza, bajo índice de escolarización 
y elevadas tasas de analfabetismo. A este analfabetismo tradicional se añade ahora el analfabetismo científico que es aún más amplio.

En resumen, sino se cumplen los Objetivos del Milenio de Naciones Unidas será muy difícil alcanzar el alfabetismo científico. El concepto de alfabetismo se expande y, junto a la alfabetización tradicional propia de la cultura letrada, emergen otros alfabetismos -relacionados con la cultura científica, tecnológica, digital e idiomática- que constituyen un prerrequisito para el ejercicio de una ciudadanía plena. Son alfabetismos que, en muchos casos, están estrechamente relacionados con la cultura letrada, pero que adquieren una identidad propia en el currículo escolar del siglo XXI. Emergen así, el alfabetismo digital, tecnológico o electrónico - digital literacy, technological literacy o e-literacy-, el alfabetismo visual -visual literacy-o el alfabetismo informacional-information literacy-, por sólo referirnos a algunos de los ejemplos más conocidos. La aparición de estos nuevos alfabetismos - digital, visual, informacional, científico, tecnológico, lingüístico...- implica en forma inexorable la eventual aparición de sus correspondientes analfabetismos. "Son sólo algunos trazos de la realidad sobre la que se proyectan las nuevas necesidades educativas y de alfabetización en la sociedad de la información." (COLL, 2005, p. 4)

\section{Principales desafíos \\ a la educación en el siglo xxi}

En la prensa diaria nos encontramos conceptos que muchos ciudadanos, más del 50\% aún en los países desarrollados y un porcentaje mayor en el mundo en desarrollo, no son capaces de entender. Entre ellos: inteligencia artificial, teoría del caos, redes neuronales, sistemas complejos, biodiversidad, nanotecnología, genoma humano, sistemas expertos, biosferas espaciales, realidad virtual, ciberespacio, teoría de supercuerdas, lógica difusa, hipótesis de gaia y otros muchos. ¿Conocemos el impacto en nuestra vida cotidiana de estas teorías y tecnologías? ¿Qué \% de las poblaciones en los países en desarrollo y desarrollados son alfabetos científicos capaces de entender estos conceptos? ¿Cómo podemos ampliar el porcentaje de alfabetizados científicos mediante la educación?

Muchas personas saben enviar emails y consultar la Web, pero no viven en la Red, les falta un cierto grado de alfabetismo al respecto. Desconocen el significado de términos como: flckr, facebook, My Space, mininova o twitter.

La educación para todos a lo largo de toda la vida es la propuesta de UNESCO para recuperar (o instaurar) el carácter de proyecto democrático 
de la educación. Para superar el analfabetismo tradicional y para construir el alfabetismo científico. Entre los principales desafíos para superar el apartheid escolar se encuentran: otorgar mayores recursos financieros a la educación; la actualización permanente de las competencias de los maestros; la puesta al día de los contenidos educativos del currículo en el marco de una educación en valores; la introducción de las nuevas tecnologías de la información y comunicación y de las redes electrónicas; la transformación de las instituciones educativas; el diseño de estrategias claras por los Ministerios de Educación; y la adecuada interrelación entre la enseñanza pública y privada y los diferentes niveles educativos y tipos de educación. Se debe insistir en el aprendizaje activo y no en la enseñanza pasiva. Es clave, en este marco, garantizar una infraestructura que otorgue a los maestros un status que prestigie socialmente la profesión y asegurar una inversión mínima en el área de las nuevas tecnologías complementada con la introducción de nuevos saberes en los contenidos. Son éstas las variables claves para una reforma de la educación, conjuntamente con la aplicación de los enunciados esenciales de la Comisión Delors: aprender a conocer, aprender a hacer, aprender a vivir juntos y aprender a ser; y la reforma del pensamiento que propugna Edgar Morin. (DELORS, 1996, p. 22-23)

Según Morin ésta "es inseparable de la reforma de la educación". Ambas deben ser construidas a través de siete saberes fundamentales que la educación del futuro debería desarrollar en cualquier sociedad y cultura, sin rechazar los usos y reglas de cada sociedad y cultura. Estos saberes son:

1) El estudio de las características cerebrales, mentales y culturales del conocimiento humano, de sus procesos y modalidades del error y de la ilusión. 2) Promover un conocimiento capaz de abordar los problemas globales y fundamentales para inscribir allí los conocimientos parciales y locales. 3) El ser humano es a la vez físico, biológico, síquico, cultural, social, histórico. Es esta unidad compleja de la naturaleza humana la que está completamente desintegrada en la educación a través de las disciplinas y que imposibilita aprender lo que significa ser humano. Hay que restaurarla de tal manera que cada uno desde donde esté tome conocimiento y conciencia al mismo tiempo de su identidad compleja y de su identidad común a todos los demás humanos. 4) Enseñar la historia de la era planetaria que comienza con la comunicación de todos los continentes en el siglo XVI sin ocultar las opresiones y dominaciones que han asolado la humanidad y que aún no han desaparecido. Habrá que señalar la complejidad de la crisis planetaria que enmarca el siglo XX mostrando que todos los humanos confrontan los mismos problemas de vida y muerte, y viven una misma comunidad de destino. 5) Las ciencias nos han hecho adquirir muchas certezas, pero de la misma 
manera nos han revelado, en el siglo XX, innumerables campos de incertidumbre. La educación debería comprender la enseñanza de las incertidumbres que han aparecido en las ciencias físicas en las ciencias de la evolución biológica y en las ciencias históricas. 6) La educación para la comprensión está ausente de nuestras enseñanzas, el desarrollo de la comprensión necesita una reforma de las mentalidades. Tal debe ser la tarea para la educación del futuro. También existe la necesidad de estudiar la incomprensión desde sus raíces, sus modalidades y sus efectos". 7) La ética no se podría enseñar con lecciones de moral. Ella debe formarse en las mentes a partir de la conciencia de que el humano es al mismo tiempo individuo, parte de una sociedad, parte de una especie. De allí se esbozan las dos grandes finalidades ético-políticas del nuevo milenio: establecer una relación de control mutuo entre la sociedad y los individuos por medio de la democracia y concebir la Humanidad como comunidad planetaria. (MORIN, 2000, p. 17-22)

Además de los principios enunciados por Delors y Morin en trabajos apoyados y difundidos por UNESCO, esta Institución lanzó en 1993 un proyecto titulado Project 2000+: Scientific and Technological Literacy for All., que tenía como objetivo esencial combatir el analfabetismo científico a nivel mundial, mediante la acción de los gobiernos con programas educativos ad hoc que construyesen los nuevos saberes necesarios en ciencia y tecnología.

\section{Alfabetismo científico, misión de la universidad y temas relevantes de investigación}

La brecha digital, científica, tecnológica e idiomática incrementa el analfabetismo tradicional y el nuevo, esto es, el analfabetismo científico. Para que se pueda aplicar el conocimiento al desarrollo, la investigación debe incluir conocimiento global y local. La investigación debe priorizar los problemas y necesidades nacionales, aunque los conocimientos globales sean prerrequisito para elaborar adecuadas políticas científicas. Lo que se necesita es aplicar a lo local los conocimientos globales, pero teniendo en cuenta lo específico, con el fin de evitar la existencia de problemas científicos huérfanos. Un grave error en muchos países ha sido aplicar soluciones importadas a los problemas locales, desde la gestión de la tierra y el agua hasta la curación de cierto tipo de cáncer.

La investigación sobre las variedades agrícolas de alto rendimiento y la implantación de prácticas agrícolas avanzadas en países en vías de desarrollo, como en el caso de la India, conllevaron la autosuficiencia de estos países en cuanto a producción de alimentos. 


\begin{abstract}
El Foro Mundial de Investigación en Salud fue creado en 1996 para intentar paliar el desequilibrio 10/90 en investigación en salud. Los estudios demostraron que el 90 \% del esfuerzo y la inversión en la investigación en salud se dedicaba a problemas que afectan solamente al $10 \%$ de la población mundial, mientras que se desatendían los problemas de salud del $90 \%$ de la población restante -principalmente en países en vías de desarrollo. Los medicamentos necesarios para prevenir o combatir las enfermedades tropicales se hallan en la categoría de medicamentos huérfanos, ya que a las empresas no les interesa asumir la investigación y el desarrollo de tales medicamentos, dado que no reportan beneficios económicos. Los problemas de salud desatendidos incluyen la tuberculosis, la malaria, la oncocercosis, la filariasis y las mordeduras de serpiente. Mientras que la tuberculosis casi ha desaparecido en las naciones desarrolladas, se ha convertido en una amenaza bastante seria en los países en vías de desarrollo, ya que ha evolucionado hacia los tipos de tuberculosis resistente a medicamentos múltiples y tuberculosis extremadamente resistente, aún más peligrosa. (SUWANWELA, 2008, p. 133)
\end{abstract}

El caso de epidemia de gripe aviar en Tailandia, Vietnam e Indonesia - en que las muestras tuvieron que ser enviadas a laboratorios de otros países para confirmar que se había desencadenado una epidemia de la enfermedad, que podía contagiarse a los humanos - muestra la importancia del desarrollo científico nacional, la importancia de invertir en las capacidades de investigación de los países en vías de desarrollo, ya que les permite enfrentarse a los problemas locales. Los laboratorios de investigación de las universidades tailandesas que estudian los genomas, la cartografía genética y las infecciones virales pudieron descodificar el virus y confirmar que efectivamente era la cepa H5N1. También en Cuba existen experiencias relevantes al respecto.

Los países en vías de desarrollo necesitan llevar a cabo una investigación esencial nacional, incluida la investigación sobre la aplicación local del fondo de conocimiento global; la investigación para comprender sus propios problemas y situación; la investigación orientada a los problemas; la investigación de políticas y de sistemas, y la investigación operativa.

Por otra parte, es cada vez mayor el debate en torno a las universidades de categoría mundial (World-Class Universities), refiriéndose este concepto a las que tienen una excelencia reconocida mundialmente (Yale, Harvard, Oxford, Cambridge, Sorbonne, Tokio...). Sin embargo, los países en desarrollo, en vez de emular para obtener indicadores muchas veces difíciles de alcanzar en sus condiciones específicas, deberían prestar más atención a un ideal de universidad que propulse el desarrollo sostenible de carácter autóctono. Más importante que 
tener los indicadores de las "world class universities" o "research universities", más importante que tener un premio Nóbel, es garantizar que existan buenas escuelas de medicina y buenos programas de formación de ingenieros agrónomos y educadores para garantizar un nivel adecuado de "capital humano y social", esto es, de recursos humanos en condiciones de generar desarrollo con equidad. (LOPEZ SEGRERA, 2007a, 2008; ALTBACH, 2006; ORDORIKA, 2008)

Vinculado a lo señalado en el párrafo anterior esta la moda de los rankings. A mediados de los 80s el Asian Wall Street Journal incluía, en una lista de las 10 mejores universidades del mundo, sólo 4 (Cambridge, Oxford, Sorbonne, Tokio) fuera de EE.UU. Según Altbach algunos de estos rankings tienen cierta respetabilidad como el de U.S. News y el del Times Higher Education Suplement (THES). En el 2005 se debatió mucho sobre la fiabilidad del ranking publicado ese año por el THES y la Shangai Jiao Tong University ${ }^{2}$. Lo que ocurre es que los ranking están construidos sobre la base de parámetros - número de Premios Nobel, profesores de la universidad con trabajos en el citation index, doctorados y maestrías, equipamiento, financiamiento...-propios de las universidades de "clase mundial" del mundo anglosajón, en especial de EE. UU. e Inglaterra y que además privilegian las ciencias duras en detrimento de las sociales y humanas.

Aplicar las normas y valores de las principales potencias académicas no medirá en forma exacta la calidad a nivel mundial, ni dará lugar a ranking mundiales de interés. En el competitivo y orientado hacia el mercado mundo académico del siglo XXI, los rankings son inevitables y probablemente necesarios. El desafío es asegurar que provean criterios exactos y relevantes y midan las cosas adecuadas. (ALTBACH, 2006, p. 80)

Otro tema relevante vinculado al alfabetismo científico es la relación entre saber ancestral y conocimiento científico moderno. Los pueblos indígenas del centro y sur de América han soportado la exclusión en todos los niveles

2 La Universidad Jiao Tong de Shangai publicó un ranking en 2004 basado en: números de Premios Nóbel, cantidad de investigadores citados en el Citation Index y la calidad de la educación en relación con el tamaño de la Universidad. Según este Estudio de las 10 mejores universidades del mundo, 8 estaban en EE.UU. encabezadas por Harvard y Stanford. En este ranking había pocas universidades fuera de EE.UU. y Europa. 9 en China, 8 en Corea del Sur, 5 en Hong Kong, 5 en Taiwán, 4 en Sudáfrica, 4 en Brasil, 1 en México, 1 en Chile y 1 en Argentina. Todas las universidades latinoamericanas aparecían en el ranking por debajo del 200 (UNAM, USP de Sao Paulo) y otras (Universidad de Chile, UniCamp, Universidade Federal do Rio de Janeiro) por debajo del 300. ¿Debemos preocuparnos por esto? ¿Debemos aspirar a lograr los indicadores de este ranking como objetivo central? Ver el ranking que publica anualmente La Universidad Jiao Tong de Shangai en: http://ed.sjtu.edu.cn/ranking.htm 
y dimensiones. Han sido sujetos a negaciones e invisibilidades jurídicas, socioeconómicas, culturales y epistemológicas, entre otras, que se evidencian en los bajos índices en los indicadores sobre el desarrollo humano, y en altos índices de pobreza. Hay entre ellos un bajo índice de alfabetismo científico. (MUÑOZ, 2008)

Existe una barrera epistemológica, es decir, un cierto tipo de "analfabetismo cultural" de amplios sectores académicos, que desde posturas monoculturales se relacionan con los pueblos indígenas, otorgándoles una condición de minoría de edad. Muchas veces, los considerados analfabetos tradicionalmente, pueden tener una actitud cultural enraizada en valores que los convierte en maestros en sabiduría ante el afán de dominio de un saber desenraizado de la ética. Por eso no deseamos que se considere que no se puede ser culto sin tener un dominio del último aporte de las TIC o del conocimiento científico.

El diálogo entre los conceptos, la experiencia y el sentido se puede sintetizar en un aforismo de los indígenas nasa del suroeste colombiano, a quienes UNESCO otorgó el título de maestros de sabiduría reconociendo así el valor del conocimiento ancestral: "La palabra sin la acción está vacía, la acción sin palabra es ciega, la palabra y la acción fuera del espíritu de la comunidad es muerte." (Muñoz, 2008, p. 108)

Por una parte, se desarrolla y consolida una educación superior propia, a partir de la historia, la cultura y el pensamiento de cada pueblo indígena y está orientada a fortalecer su identidad. Se destacan en este tipo las escuelas de derecho propio en Ecuador, Colombia y Bolivia, la Escuela de Medicina Kallaguaya de Bolivia y el proyecto Kawsy Unik de los pueblos indígenas de los países andinos. (MUÑOZ, 2008, p. 109)

Por otra, están las experiencias de educación superior bilingüe e intercultural desarrolladas por los pueblos indígenas. Tales como: la Universidad de las Regiones Autónomas de la Costa Nicaragüense, URACAN, y la Universidad Autónoma Intercultural del Consejo Regional Indígena del Cauca, Colombia (UAIN). (MUÑOZ, 2008, p. 109)

Nos enfrentamos a varios retos:

a) el de cambiar la forma de reconocer pueblos, culturas y saberes desde un enfoque monocultural;

b) el reto de superar la colonialidad del saber y el mimetismo respecto a Europa y Estados Unidos;

c) el del diálogo entre la ciencia y el conocimiento ancestral;

d) y el reto de construir universitas, como espacio "donde sea posible el encuentro y el diálogo de las ciencias, los conocimientos, los saberes, las culturas, los pueblos y las naciones. (MUÑOZ, 2008, p. 110) 


\section{Conclusiones y recomendaciones}

Para construir el alfabetismo científico pudiéramos sugerir algunas recomendaciones en el plano de la educación y de las TIC:

a) Desarrollar la educación permanente para todos durante toda la vida para formar a los usuarios de las «autopistas de la información», y una pedagogía del ejemplo, de la transformación de la información en conocimiento.

b) Facilitar el acceso universal a las TIC con el objetivo de garantizar el derecho de todo individuo a «investigar y recibir informaciones y opiniones, así como a difundirlas, sin límites de fronteras, por cualquier medio de expresión» (Declaración Universal de los Derechos Humanos, artículo I9).

c) Estimular «la cooperación intelectual de todas las naciones en todas las ramas de la actividad intelectual» (Constitución de la UNESCO), interconectando en el plazo de una generación, a través de Internet, las escuelas, universidades, bibliotecas y museos de todo el mundo.

d) Desarrollar proyectos de cooperación Norte-Sur en materia de tecnologías de la información.

Para concluir, debemos recomendar la puesta en práctica de los principios y enunciados de la Declaración Final de la Conferencia Regional de Educación Superior de UNESCO (CRES, 2008) preparatoria de la Conferencia Mundial de Educación Superior (CMES+10) y de su Plan de Acción, como instrumentos claves para el desarrollo del alfabetismo científico. ${ }^{3}$

Los valores y principios de la Declaración de la CRES 2008, particularmente el concepto de educación como bien público social, derecho universal y deber del Estado, obligan a la Educación Superior (ES), independientemente de la naturaleza jurídica de las IES, a:

- afirmar la noción de calidad vinculada a la pertinencia e inclusión social;

- formar y promover una cultura democrática y ciudadana, en colaboración con los niveles previos de educación.

- afirmar los valores humanísticos y de la promoción de la cultura de Paz,

3 Consultar la Declaración Final de la CRES 2008 y su Plan de Acción en: http://www.google.es/search $? \mathrm{hl}=\mathrm{es} \& \mathrm{q}=\mathrm{CRES}+2008+$ plan + de + accion $+\mathrm{y}+$ declaracion + final $\&$ btnG $=$ Buscar\&meta $=$ 
el respeto y valorización de la diversidad cultural y el compromiso con el desarrollo humano y sustentable;

- generar condiciones para un diálogo entre pares con otras regiones del planeta con énfasis en la cooperación sur-sur;

- contribuir a la solución de las problemáticas sociales más agudas y al cumplimiento de los objetivos del milenio.

En esta perspectiva se elaboró un Plan de Acción con cinco lineamientos principales:

a) Impulsar la expansión de la cobertura en educación superior, tanto en pregrado como en postgrado, con calidad, pertinencia e inclusión social;

b) promover políticas de acreditación, evaluación y aseguramiento de la calidad;

c) fomentar la innovación educativa y la investigación en todos los niveles;

d) construir una agenda regional de Ciencia, Tecnología e Innovación para la superación de brechas y para el desarrollo sustentable de ALC, acorde a las políticas generales de cada Estado miembro;

e) propugnar la integración regional latinoamericana y caribeña y la internacionalización de la educación superior en la región mediante, entre otras iniciativas, la construcción del ENLACES - Espacio de Encuentro de América Latina y el Caribe para la Educación Superior. 
Referencias

ALTBACH, P. International higher education. Reflections on policy and practice. Boston: Boston College CIHE, 2006.

AAAS. Science for all Americans. A project 2061 report on literacy goals in science, mathematics and technology. New York: American Association for the Advancement of Science, 1989.

BROVETTO, J. La educación superior para el siglo XXI. En: TÜNNERMANN, C.; LOPEZ SEGRERA, F. La educación en el horizonte del siglo XXI. 2000. Caracas: Ediciones IESALC/UNESCO. Colección Respuestas n. 12.

BRUNNER, J. J. G. ELACQUA. Informe Capital Humano en Chile. Santiago de Chile: Escuela de Gobierno, Universidad Adolfo Ibáñez, 2003. Disponible en: <http://www.uai.cl/p4_home/site/asocfile/ ASOCFILE120030528134519.pdf>. Acceso en: 5 oct. 2008.

BRUNNER, J. J. Higher education in Latin America and the Caribbean. 2004. Diponible en: <http://mt.educarchile.cl>. Acceso en: 7 oct. 2008.

BRUNNER, J. J. Tendencias recientes de la educación superior a nível internacional. 2005. Diponible en: <http://mt.educarchile.cl>. Acceso en: 9 oct. 2008.

BRUNNER, J. J. Documentos varios elaborados para el curso impartido en el Centro Universitario Salesiano de São Paulo. 2006. Diponible en: $<$ http://mt.educarchile.cl>. Acceso en: 1 oct. 2008.

BYBEE, R. Toward and Understanding of Scientific Literacy. En: GRÄBER, W., BOLTE C. California: Scientific Literacy. 1997. Kiel, IPN, 37-68.

CABRAL, Perdomo Ignacio. Alfabetismo científico y educación. Instituto Tecnológico y de Estudios Superiores de Monterrey Campus Central de Veracruz, 2001. Disponible em: <www.rieoei.org/deloslectores/Cabral. PDF>. Acceso en: 10 oct. 2008.

CEPAL; UNESCO. Educación y conocimiento: eje de la transformación productiva con equidad. Libros de la CEPAL, Comisión Económica para América Latina y el Caribe (CEPAL) y Oficina Regional de Educación de la UNESCO para América Latina y el Caribe, Santiago de Chile, Agosto, 1992. 
CEPAL. Anuario estadístico de América Latina y el Caribe. 2007. Disponible en: <http://www.cepal.org>. Acceso en: 10 oct. 2008.

CINDA. Educación superior en Iberoamérica. Informe 2007. Santiago de Chile, 2007.

CINDA; Cátedra UNESCO de Dirección Universitaria (CUDU).

Acreditación y dirección estratégica para la calidad de las universidades. Santiago de Chile, 2007.

COLL, C. Lectura y alfabetismo en la sociedad de la información. UOC Papers n. 1, 2005. UOC. <http://www.uoc.edu/uocpapers/1/dt/esp/coll.pdf> CORTADELLAS, J. Dirección estratégica y calidad total, ¿también en lãs universidades?" En CINDA; Cátedra UNESCO de Dirección Universitaria (CUDU) (2007) Acreditación y dirección estratégica para la calidad de las universidades. Santiago de Chile, 20007.

DE LA VEGA, I., VESSURII, H. El uso de la cienciametría en la construcción de las políticas tecnocientíficas en América Latina, uma relación incierta. En JORNADAS LATINOAMERICANAS DE ESTUDIOS SOCIALES DE LA CIENCIA Y LA TECNOLOGÍA, 6., Esocite, 2006, Bogotá, 2006.

DELORS, J. La Educación encierra un tesoro. Paris, UNESCO, 1996.

DIAS SOBRINHO, J. Dilemas da educação superior no mundo globalizado. São Paulo: Casa do Psicólogo, 2005.

DIDOU, S. Internacionalización y proveedores externos de educación superior en América Latina y el Caribe. México D.F: ANUIES, 2005.

DIDRIKSSON, A. The role of higher education for human and social development in Latin America and the Caribbean. In: GUNI. Higher education in the World. 3., New.York: Palgrave Macmillan, 2008.

EFA. Global monitoring report. Paris: UNESCO, 2007.

ESCOTET, M. A. Gobierno, rendición de cuentas y financiamiento de la universidad. En: En La educación superior en el mundo 2006: la financiación de las universidades. GUNI. Madrid: Ediciones Mundipresa, 2006.

FERNÁNDEZ LAMARRA, N. Una perspeciva comparada de la educación superior en América Latina. Ponencia presentada a Universidad 2008, Habana, 2008. 
FUENTE, DE LA, J. Las universidades, la inteligencia de los países. Foreign Affairs en español, Mexico-DF, v.. 5, n. 2, 2005.

GAZZOLA, A. L. Integración académica e internacionalización de la educación superior. CONFERENCIA EN UNIVERSIDAD 2008, Habana, 2008.

GENTILI, P.; FRIGOTTO, G. La Ciudadanía negada. Buenos Aires: CLACSO, 2001.

GUNI. La educación superior en el mundo 2006: la financiación de lãs universidades. GUNI. Madrid-Barcelona-México: Ediciones Mundipresa, 2006a.

GUNI. La Educación Superior en el Mundo 2007. Acreditación para la garantía de la calidad:¿Qué está en juego?. GUNI. Madrid-BarcelonaMéxico: Mundi-Prensa, 2006b.

GUNI. Higher education in the World 3. New York: Palgrave Macmillan, 2008.

INVERNIZZI, N. Participación ciudadana en ciencia y tecnología: desafios en el contexto latino-americano. En: JORNADAS LATINOAMERICANAS DE ESTUDIOS SOCIALES DE LA CIENCIA Y LA TECNOLOGÍA, 5., Esocite, México: Toluca, 2004.

KUHN T. La estructura de las revoluciones científicas. Madrid: Fondo de Cultura Económica,1997.

KLIKSBERG, B. Inequidad en la educación en América Latina algunas cuestiones estratégicas. Biblioteca Digital, Iniciativa Interamericana de Capital Social Etica y Desarrollo, 2002. Disponible en: <www.iadb.org/ etica $>$.

LOPEZ SEGRERA, F.; TÜNNERMANN, C. La Educación en el horizonte del siglo XXI. Colección Respuestas, Caracas, n. 12. UNESCO, 2000.

LOPEZ SEGRERA, F. Globalización y educación superior en América Latina y el Caribe. Colección Respuestas, Caracas, n. 18. IESALC/ UNESCO, 2001

LOPEZ SEGRERA, F.; MALDONADO, A. Educación superior Latinoamericana y organismos internacionales. Un análisis crítico. Prefacio Philip G. Altbach. UNESCO, Boston: College; Cali, Colombia: Universidad de San Buenaventura de Cali, 2002. 
LOPEZ SEGRERA, F. Educación Permanente, calidad, evaluación y pertinencia. UNESCO; Cali, Colombia: Universidad de San Buenaventura, 2002.

LOPEZ SEGRERA, F. Higher education and research in the Latin American Region. UNESCO FORUM. ED.04/Conf.611/35. Paris 22 nov. 2004a

LOPEZ SEGRERA, F. Posibles escenarios mundiales de la educación superior. Perfiles Educativos, n. 109-110, CESU, UNAM, 2005 a.

LOPEZ SEGRERA, F. Análisis prospectivo de la educación superior a nível mundial. Revista Paraguaya de Sociología, Asunción, v. 42, n. 124, 2005b.

LOPEZ SEGRERA, F. El enfoque sobre los conceptos de calidad y acreditación en la CMES y en las reuniones de seguimiento. En LA EDUCACIÓN SUPERIOR EN EL MUNDO 2007. Acreditación para la garantía de la calidad: ¿Qué está en juego? GUNI, Madrid: Ediciones Mundipresa, 2006a.

LOPEZ SEGRERA, F. La educación superior en el Caribe. EN LA EDUCACIÓN SUPERIOR EN EL MUNDO 2006: la financiación de las universidades. GUNI, Madrid: Ediciones Mundipresa, 2006b.

LOPEZ SEGRERA, F. América Latina y el Caribe: principales tendencias de la educación superior. Avaliação, Campinas; Sorocaba, v. 11, n. 3, sep. 2006c.

LOPEZ SEGRERA, F. Escenarios mundiales de la educación superior. Buenos Aires: CLACSO, 2006d.

LOPEZ SEGRERA, F. Educación superior internacional comparada: escenarios, temas y problemas. 2007. Disponible en: <http://www.brunner. $\mathrm{cl} />$. 2007a.

LOPEZ SEGRERA, F. Escenarios mundiales y regionales de la educación superior. Avaliação, Campinas; Sorocaba, v. 12, n. 03, nov. 2007b.

LOPEZ SEGRERA, F.; SANYAL, B.C An overview of regional perspectives on the role of higher education in social and human development. In: GUNI, Higher Education in the World 3., New York: Palgrave Macmillan, 2008.

MACEDO, B., KATZKOWICZ, R. Alfabetización científica y tecnológica. Aportes para la reflexión. Disponible en: $<\mathrm{http}$ ://www.unesco.cl/medios/ alfabetizacion_cientifica_tecnologica_aportes_reflexion.pdf?menu=/esp/ biblio/docdig/> en OREALC/UNESCO, Santiago Chile, 2000. 
MARCHESI, A. Un proyecto educativo iberoamericano. Fundación Carolina, Madrid, 2007. Disponible en: <http://www.google.es/search?hl=es \&q=Un+proyecto+educativo+iberoamericano+Alvaro+Marchesi\&btnG=Bus car+con+Google\&meta=>.

MAYOR, F.; BINDÉ, J. Un mundo nuevo. Barcelona: Ediciones UNESCO, 2000a.

MAYOR, F. ; TANGUIANE, S. L'enseignement supérieur au XXIe siécle. Paris: Hermes Science Publications, 2000.

MOLLIS, M. (compiladora). Las universidades en América Latina: ¿̇reformadas o alteradas? Buenos Aires : CLACSO, 2003.

MORIN, E. Los siete saberes necesarios a la educación del futuro. Caracas: UNESCO-IESALC, 2000.

MUÑOZ, M. R. Retos a la educación superior en el centro y sur de América desde el diálogo entre ciencia y conocimiento ancestral. En: La educación superior en el mundo 3. GUNI. Madrid: Mundi-Prensa, 2008.

OCDE. Annual Report. 2007. Disponible en : <www.OCDE.org/ bookshop>.

OLIVA, J.; ACEVEDO, J. A. La enseñanza de las Ciencias en Primaria y Secundaria hoy. Algunas propuestas de futuro. Revista Eureka sobre Enseñanza y Divulgación de las Ciencias, v. 2, n. 2, p. 241-250, 2005. Disponible en: <http://www.apac-eureka.org/revista> .

ORDORIKA, I. Contemporary challenges for public research universities. In: GUNI, Higher education in the world. 3. New York: Palgrave Macmillan, 2008.

PARKER GUMUCIO, C. Sociedad de la Información, Investigación y Desafíos a la Educación Latinoamericana y Chilena. Revista de Sociología, Universidad Católica Cardenal Silva Henríquez, 2006.

PARKER GUMUCIO, C. Science and Technology in University Student's Worldview. A study in a developing society shaped by globalization: the Chilean case. 2006. WORLD CONGRESS OF SOCIOLOGY, Durban, South Africa, 23-29 Jul. 2006.

PARKER GUMUCIO, C. Orientaciones hacia la ciencia y la tecnología en los estudiantes universitarios de hoy resultados de una encuesta. Contribuciones Científicas y Tecnologías, 2006. 
PARKER GUMUCIO, C. Por una nueva cultura científica: más allá de lãs dos culturas. Este trabajo forma parte del proyecto Fondecyt No 1070172 , Alfabetismo científico para el Chile del mañana: En busca de una definición conceptual y operacional para evaluar la formación de los Ciudadanos Del Mañana, 2008.

POLINO, C. El último eclipse total de Sol del milenio: ciencia e ignorância científica. JORNADAS INTERNACIONALES DE COMUNICACIÓN, 14. Universidad de Navarra, España, 1999. Disponible en: <http://www. unav.es/ fcom/jornadas/u.htm>.

POLINO C., FAZIO M.; VACAREZZA L. Medir la percepción pública de la ciencia en los países iberoamericanos. Aproximación a problemas conceptuales. Revista Iberoamericana de Ciencia Tecnología, Sociedad e Innovación, n. 5, jan./abr. 2003.

PRIE. Cumbre de las Américas. Panorama educativo 2005: progresando hacia las metas, Proyecto Regional de Indicadores Educativos. UNESCO y Secretaría de Educación Pública de México, 2005. Disponible en: <www. unesco.cl_medios_biblioteca_documentos_panorama_educativo_2005_ progresando_hacia_las_metas.pdf $>$. Programa de las Naciones Unidas (2006). Las tecnologías: ¿um salto al futuro. Informe de desarrollo Humano 2006 Santiago Chile.

PNUD. Human Development Report. 2008. Dsiponible en: <http://www. undp.org>.

SUWANWELA, C. La colaboración política y social de la investigación. En: La educación superior en el mundo 3. GUNI. Madrid: Mundi-Prensa, 2008.

TÜNNERMANN BERNHEIM, C. La universidad necesaria para el siglo XXI. Managua: HISPAMER/UPOLI, 2007.

UNESCO. Conferencia Mundial sobre Ciencias y el Uso del Conocimiento Científico. Budapest: UNESCO-ICSU, 1999.

UNESCO. Ética de la Ciencia y la Tecnología. Contacto, Boletín Internacional de la Unesco de Educación Científica, Tecnológica y Ambiental, v. XXIX, n. 3-4, 2004. Disponible em: <http://www.unesco.org/ education/educprog/ste/index.html>.

UNESCO. Global education digest. Montreal: UIS, 2007.

WORLD BANK. Higher education. The lessons of experience. Washington, D. C., 1994 
WORLD BANK. Higher education in developing countries: peril and promise.Washington, D.C., 2000.

WORLD BANK. Constructing knowledge societies: new challenges for tertiary education. Washington, D.C., 2002.

ZICCARDI, A. Pobreza, desigualdad social y ciudadanía. Buenos Aires: CLACSO, 2001.

\section{Websites}

http://portal.unesco.org http://www.iesalc.unesco.org.ve/ http://unesdoc.unesco.org http://www.uis.unesco.org http://www.OCDE.org http://www.worldbank.org http://www.iadb.org http://cepal.org http://www.undp.org http://www.universia.net http://www.unesco.org/aiu http://www.clacso.org http://portal.unesco.org/education/en/ev. Universidad de las Naciones Unidas (UNU). http://www.unu.edu http://www.fiuc.org/ http://www.crue.org http://www.cinda.cl http://www.brunner.cl http://www.educarchile.cl http://www.bc.edu/cihe/ http://www.unam.mx/coordhum/riseu http://www.usp.br/nupes/. http://www.gatswatch.org http://www.guni-rmies.net http://www.upc.es/cudu http://www.uoc.edu/portal/english/index2.html 